\title{
Balancing Energy Harvesting and Transmission Scheduling in Aggregation Convergecast
}

\author{
Jesse Huard \\ Department of Computing Science \\ University of Alberta \\ Edmonton, Alberta, Canada T6G 2E8 \\ jhuard@ualberta.ca
}

\author{
Ioanis Nikolaidis \\ Department of Computing Science \\ University of Alberta \\ Edmonton, Alberta, Canada T6G 2E8 \\ nikolaidis@ualberta.ca
}

\begin{abstract}
We study tradeoffs between aggregation convergecast and energy harvesting in wireless sensor networks. Existing aggregation convergecast algorithms do not capture the volatile nature of energy reserves of energy harvesting nodes. We therefore propose, and evaluate, new scheduling schemes to address this gap. We also introduce metrics to capture the impact of the inevitable energy depletion on the quantity of aggregated data received at a sink node. Specifically, we consider node behaviors where the inability to perform prompt communication due to energy depletion results in a reduction of the sampling rate (including for aggregated data) and, if it persists, loss of data. The performance evaluation is based on heat flow data collected from an apartment building in the Canadian North. The collected heat flow data are used to approximate the energy harvesting output of thermoelectric harvesters.
\end{abstract}

\section{CCS CONCEPTS}

- Networks $\rightarrow$ Sensor networks; In-network processing; Logical / virtual topologies; • Hardware $\longrightarrow$ Energy generation and storage;

\section{KEYWORDS}

aggregation convergecast scheduling; energy harvesting; wireless sensor networks

\section{ACM Reference Format:}

Jesse Huard and Ioanis Nikolaidis. 2018. Balancing Energy Harvesting and Transmission Scheduling in Aggregation Convergecast. In 21st ACM International Conference on Modelling, Analysis and Simulation of Wireless and Mobile Systems (MSWIM '18), October 28-November 2, 2018, Montreal, QC, Canada. ACM, New York, NY, USA, 9 pages. https://doi.org/10.1145/3242102. 3242132

\section{INTRODUCTION}

A common traffic pattern in wireless sensor networks (WSNs) is to gather sensor data generated by the network at a centralized sink node [5]. When gathering this data, we are often interested in producing in-network some kind of aggregate measurement from the data. For example, we might be interested in the maximum,

Permission to make digital or hard copies of all or part of this work for personal or classroom use is granted without fee provided that copies are not made or distributed for profit or commercial advantage and that copies bear this notice and the full citation on the first page. Copyrights for components of this work owned by others than the author(s) must be honored. Abstracting with credit is permitted. To copy otherwise, or republish, to post on servers or to redistribute to lists, requires prior specific permission and/or a fee. Request permissions from permissions@acm.org.

MSWIM '18, October 28-November 2, 2018, Montreal, QC, Canada

(c) 2018 Copyright held by the owner/author(s). Publication rights licensed to ACM. ACM ISBN 978-1-4503-5960-3/18/10 . \$ \$15.00

https://doi.org/10.1145/3242102.3242132 minimum, or top- $k$ values or in a $k$-bin histogram over a given time interval. When this is the case, we can aggregate sensor data as it is forwarded along the path towards the sink. This traffic pattern is called aggregation convergecast. To achieve operation of the network, we construct a collision-free time division multiple access (TDMA) transmission schedule which describes when nodes should transmit their aggregated data. The construction of an aggregation convergecast schedule induces a spanning tree over the network topology. The objective of aggregation convergecast scheduling is to minimize the length of the schedule [4]. Unfortunately, minimum length schedules are usually not energy-efficient, in the sense that they can result in fast energy depletion of "bottleneck" nodes.

In an energy harvesting wireless sensor network (EH-WSN), nodes continually gather energy from ambient sources of energy such as solar, wind, or thermal energy [8]. Harvested energy is stored, e.g., in a supercapacitor [10], until the node's controller requires the energy to perform computation or communication. Outside of these brief periods of communication, we usually keep the nodes' communication equipment unpowered. This duty cycling allows us to preserve energy on the network nodes. A goal in $\mathrm{EH}-$ WSNs it to choose the duty cycle and scheduling of tasks in the network so as to achieve perpetual operation of the network. Nodes should not attempt to consume more energy than is available so as to achieve energy neutral operation, resulting in long, autonomous, operational lifetime.

Generally, existing algorithms for aggregation convergecast do not take the energy constraints of network nodes into account and assume that nodes are fully powered [5]. If used naively as part of an EH-WSN deployment, these algorithms may cause load imbalances between the nodes resulting in energy depletion and thus missing data from our aggregate measurements. Similarly, energy aware aggregation tree scheduling algorithms exist, but they assume finite non-replenishable energy levels at each node, which is not true in an EH-WSN $[9,12]$. In this paper, we study specialized energy harvesting aware aggregation convergecast scheduling algorithms.

Further, beyond creating a schedule, we would like to be able to characterize how much data is missing from our aggregate measurement when node failures or energy violations do occur. This allows for graceful degradation of the network as we can express our confidence in the received data and can tell how many nodes the aggregate accounts for. We will attempt to accomplish this by taking advantage of our aggregation convergecast traffic pattern to piggy-back additional data completeness metrics along with our aggregation messages.

Our performance results based on real measurements of heat flow and hence on thermoelectric harvesting output from an apartment 
complex in Fort McMurray, Alberta, Canada [6]. We use historical data from this deployment gathered through the calendar years of 2013 and 2014. The data set from 2013 is used to inform our energy prediction models (e.g. model fitting where necessary), while the year of 2014 data is used in its source form to produce predictions to construct future aggregation convergecast schedules. The apartment building consists of 12 separate apartments, with 4 floors of 3 apartments each.

The rest of the paper is structured as follows: Section 2 summarizes the immediately relevant related work, and the scheduling scheme family that we extended. Section 3 provides the many facets of the system model: its graph communication abstraction, the energy harvester, the energy buffer, and the communication controller energy consumption. Section 4 introduces the metrics we used to characterize the (possibly challenged) completeness of the collected data due to sensor outages. Section 5 outlines the evaluated scheduling schemes. Section 6 provides the performance evaluation model parameters and the derived results. We conclude with Section 7 summarizing the contributions and possible future directions.

\section{RELATED WORK}

Energy harvesting is a particularly challenging mode of operation in aggregation convergecast. If we attempt to adhere to a schedule which causes energy violations, i.e., insufficient energy to implement the stipulated transmissions, we will be missing data at the sink. If the nodes with energy violations have many descendants in the aggregation convergecast tree, this could cause a large portion of data (from all nodes of a subtree) to be missing. Existing aggregation convergecast schemes do not take into account the energy constraints of nodes. Instead, they attempt to minimize the length of the schedule, which is known as the "Minimum Data Aggregation Time" (MDAT) problem. MDAT has been proven to be NP-hard, so existing algorithms are usually of a heuristic $[4,5]$ nature. The lack of energy efficiency objectives is likely to introduce energy violations - a fact that is confirmed in the performance analysis section.

A particular variety of algorithms address the question of maximum lifetime aggregation tree scheduling for nodes with finite energies. Assuming some initial level of energy available at each node, they construct a schedule which maximizes the time until the first node failure due to energy depletion. This is achieved by defining some sort of load function, and then attempting to balance the load between nodes in the network. The particular variety of formulations is related to other hard problems on spanning tree construction, such as the minimum degree spanning tree problem and the bounded degree spanning tree problem [12]. As such, it has been proven that constructing a longest lifetime schedule is NPhard [9]. As in all cases of aggegation convergecast, it is assumed that messages are of fixed size, and the communication energy costs are $E_{t x}$ and $E_{r x}$ to transmit or receive respectively. Further, the closest performance an approximation algorithm can reliably achieve is building a schedule with lifetime less than or equal to $\left(\frac{1}{l^{*}}+\frac{E_{r x}}{E_{m}}\right)^{-1}$ unless $\mathrm{P}=\mathrm{NP}$ [9]. In the preceding equation, $l^{*}$ is the maximum achievable network lifetime, and $E_{m}$ is the least energy among all nodes.
An existing approximation scheduling algorithm for the maximum lifetime network which meets the theoretical performance bounds described in [12] and works by taking an arbitrary spanning tree and then iteratively making 'improvements' to it until it is no longer able to do so. At any point in the algorithm, the schedule is valid and can be returned. The improvements are made by adding a non-tree edge to the tree, forming a cycle. We then attempt to reduce the degree of a 'bottleneck' node on the cycle by removing one of its incident edges. This results in a transfer of one degree between this node and another non-'bottleneck' node. We identify 'bottlenecks' by defining a load function over the current degree $x$ of the node $v$ in the tree, $f_{v}(x)=\frac{(x-1) E_{r x}+E_{t x}}{E_{v}}$, where $E_{v}$ is the current energy. For the sink node, we set the load function to $f_{v}(x)=0$, which accounts for the fact that the sink is fully powered and therefore has no energy constraints. This algorithm has a running time of $O(|E||V| \alpha(|E||V|))$, where $\alpha$ is the inverse Ackerman function.

In Section 5 we extend [12], noting that the schedule it produces is valid and, potentially, useful in the extreme case that no energy harvesting occurs. Technically, we will be, possibly for every reporting period, adjusting the schedule by iterating over the bottleneck identification and improvement of [12], albeit using different estimates for the harvested energy reserves.

\section{SYSTEM MODEL}

We adopt a model similar to that in the existing literature [5, 7, 9, 12]. We assume a fixed communication graph, $G=(V, E)$, which has a designated sink node $v_{\operatorname{sink}}$ which is fully powered. We adopt a constant reporting cycle/round, after which nodes will attempt to report to the sink via an aggregation convergecast schedule. We assume synchronization between nodes, and that nodes follow a synchronized duty cycle, i.e., they begin executing the schedule during the duty cycle activation. We assume that network wirelress capacity is not a bottleneck to aggregation convergecast; as the amount of data sent is usually small compared to available bitrates. Transmissions are assumed received if there is no collision at the intended receiver.

We consider a message to have been sent or received if the node remains operational until the message has been fully communicated. We assume that the sender knows if the receiver has successfully received the message, which could be implemented via a simple ACK mechanism. We omit the energy requirements for sensing and computation. This is not unreasonable, as the energy cost for communication tends to dominate the overall energy expenditure of the nodes. Further, we do not consider the costs associated with schedule transmission. We assume that after the sink has computed a schedule all other nodes know their slot position, but do not make further use of the schedule. As we will discuss in the conclusions, it may be possible to make local node decisions based on knowing some portion of the schedule. If we were to attempt this, we would then need to consider the communication cost of distributing the schedule.

\subsection{Energy Modelling}

Energy Harvester: We use a fairly straightforward model for our energy harvester. We assume that when there is an available 
power flux, it can be harvested. This applies to harvesters such as thermoelectric generators and photovoltaic panels. We then define our harvester according to the following characteristics:

- Area $A\left(\mathrm{~m}^{2}\right)$ : The physical area of the harvester.

- Efficiency $\eta(\%)$ : The power conversion efficiency of the harvester.

- Internal Resistance $R(\Omega)$ : The internal resistance of the harvester, used to calculate output voltage.

Given an available flux $j$, the output power of the harvester is $P=j * A * \eta$. This can then be easily converted to input energy for the energy buffer model by multiplying by a time step. We can calculate the voltage as $V=\sqrt{P * R}$.

Energy Buffer: Our current model implicitly assumes that we are using a supercapacitor as our energy buffer. We define our supercapacitor based on the following characteristics:

- Capacitance $C(F)$ : The capacitance of the supercapacitor.

- Leakage Current $I(A)$ : The constant leakage current of the supercapacitor.

- Maximum Stored Energy $E_{\max }(J)$ : The maximum amount of energy we can practically store in the supercapacitor.

We use a slightly modified Energy Iteration Equation (EIE) supercapacitor model for simulating the supercapacitor [11]. The EIE model works by calculating changes in the energy buffer over discrete time steps of interval $\Delta t$. Given the energy level $E_{t}$ of the buffer at time $t$, we can calculate the new energy as: $E_{t+1}=$ $E_{t}+E_{\text {in }}-E_{\text {out }}-P * \Delta t . E_{\text {in }}$ is the energy harvested over the last time period, $E_{\text {out }}$ is the energy consumed, and $P$ is the leakage power in Watts. We can calculate this power as $P=I * V$, where $V$ is the current voltage, $V=\sqrt{2 * E_{t} / C}$ This voltage is also used to inform the communication controller model (described next) in order to determine when the controller is non-operational.

Our model differs slightly in that we assume a constant worstcase leakage current, as opposed to an energy dependent leakage current, which requires profiling of the physical supercapacitor. We also assume a maximum practical stored energy, which we take from the supercapacitor datasheet. This adds a ceiling to the energy level of the supercapacitor when using the EIE model.

Communication Controller: We define our communication controller based on the following characteristics:

- Transmission Speed $S$ (kbps): The speed at which our controller can transmit data.

- Transmission Current $I_{t x}(A)$ : The current draw of the controller radio during transmission.

- Reception Current $I_{r x}(A)$ : The current draw of the controller radio during reception.

- Operating Voltage $V_{\text {oper }}(V)$ : The voltage at which the controller operates.

- Cut-off Voltage $V_{\text {cutoff }}(V)$ : The voltage below which the controller stops operation, i.e., the transceiver goes "dark".

Given a message with a constant size of $M$ kilobits, we can derive the energy cost per message for transmission and reception. The time to transmit the message is simply $t=M / S$. We can derive the power draw of transmission and reception as $P_{t x}=I_{t x} * V_{o p e r}$ and $P_{r x}=I_{r x} * V_{o p e r}$. Then, the energy cost is $E_{t x}=P_{t x} * t$ and
$E_{r x}=P_{r x} * t$. Using these energy costs, we can quantize the number of messages that a node is capable of receiving or transmitting. The cut-off voltage links the controller to the energy buffer and allows us to determine when the controller becomes non-operational. If the voltage provided by the buffer is below the cut-off, we consider the controller to be unable to operate.

\section{DATA COMPLETENESS METRICS}

We develop a straightforward aggregation algorithm for characterizing the amount of data received at the sink node during each reporting period. Our algorithm allows us to quantify the amount of sensor data we are missing over the reporting period, as well as during what times, and also the number of nodes contributing to the report.

We employ a simple sub-sampling strategy with the sub-sampling by a factor which is a power of two. For example, assume that each (leaf) node collects report_period/report_resolution samples during the periodic report interval. If it cannot transmit them out due to energy depletion, it can sub-sample the first round of collected data by a factor of 2, making space for storing the next round data (sub-sampled also by a factor of 2). If it again cannot transmit, the original data, and the data of the second round are further subsampled by a factor of 2 (hence by a total factor of 4 ) and the new data are sub-sampled by a factor of 4 . (Notice that the next round of data, if still unable to transmit, can be stored in the space made by the previous sub-sampling.) For the sake of convenience the number of samples that can be contained in a report message is assumed to be a power of two, i.e., $2^{k}$. Consequently, sub-sampling, once we reach $k$-fold sub-sampling, i.e. by a factor $2^{k}$, reduces an entire data collection round to a single element. At this point, (older) sub-sampled data would need to be dropped. Technically, it is probably more desirable to drop old data after a certain level (less than $k$ ) of sub-sampling has been reached. For example, in our performance study we drop older samples after we have sub-sampled by a factor of 4 and still cannot transmit the data report.

Additionally, in aggregation convergecast, we also need to keep track of how many nodes (under a subtree) have provided input in an aggregation report, and, more crucially, how an interior (nonleaf node) combines sub-sampled data that eventually make it from one or more of its children with its own (possibly also sub-sampled) data. The strategy we take is that an interior node sub-samples the data it collects to the worst of all sub-sampling factors across the data it aggregates. Furthermore it keeps track of the count of all children (sub-trees) that contribute in a possibly sub-sampled report. To this end, we annotate the period aggregation reports with the following information:

- Latest Time: The timestamp of the latest update to the report.

- Age: The age of the report in number of reporting cycles elapsed up to its transmission (also represents the number of reporting cycles this report covers).

- Sub-sampling factor: How many reporting periods our report is capable of containing information for (power of two, starting with $2^{0}=1$ ).

- Samples: The list of actual samples (possibly sub-sampled) of total length up to $2^{k}$. 
- Counts: A list of integers that represent the number of nodes the report accounts for at each reported cycle combined in the report.

The list of counts, age, and sub-sampling factor come into play when a node fails to have its message received at the next node. When this occurs, the node increments its age by one, and if it is now larger than the resolution, multiplies the sub-sampling factor by two. If the sub-sampling increases, the node will reduce its samples accordingly.

The operator of the network decides on a report resolution which determines the number of samples in a period, based also on sensor node storage constraints. A maximum report lifetime is also decided for when old data are dropped and, also, what will be the length of the count list. For simplicity, we restrict the report resolution to a power of two, and ensure that this is greater than or equal to the maximum lifetime raised to the power of two.

Note that interior nodes have to aggregate and forward data at the worst (highest) sub-sampling across their children nodes (plus their own collected data) because they have no way to make up for the missing data that were discarded because of sub-sampling. The process we describe is generic and results in loss of accuracy. Nevertheless, it might be useful to consider application-specific alternatives to blindly sub-sampling. An example is, if the aggregation is for the average across an entire network, we can subsample by means of filtering, i.e., replacing every two samples by their average, etc. and then (in aggregating) by calculating weighted averages by accounting for how many nodes have reported a certain value as being the average across all of them.

Overall, the described approach allows us to attempt a 'besteffort' data delivery even after several communication failures. If a node is unable to transmit for one reporting period, it may still be able to have its data received at the sink eventually. Putting a maximum lifetime on the age of the data allows us to limit the loss of information due to sub-sampling. If the operator of the network is not interested in old data, they can use a more aggressive maximum lifetime where old data is dropped sooner. Similarly, if this data is important it can be held for longer. In addition, since the maximum size of the report messages does not depend on the number of nodes in the network, this algorithm should scale to large network sizes.

Note that there is also a consideration to be made here for the actual sensor data aggregates received at the sink. In our current implementation, we assume that the new aggregate accounts for the total lifetime of the message. For example, if the message has an age of 3, then the aggregated data correspond to the last three reporting periods. This results in some loss of granularity of the data received at the sink. If this is undesirable, it may be possible to also maintain a limited amount of historical data, similar to the counts list but this would entail additional overhead, or would necessitate variable-sized messages. Such extensions are left for future work.

\section{SCHEDULING ALGORITHMS}

\subsection{MINT}

This scheduler builds a static schedule using the "Minimum Interference Network Topology" (MINT) heuristic [5]. This is a heuristic which is known to give state of the art performance on the MDAT problem. We choose to include this scheduling algorithm since it provides a good baseline for the performance of scheduling algorithms that lack harvesting-aware capability.

As noted earlier, we also extend [12], which we call, Longest Lifetime $(L L)$ by considering different ways in which estimates for the energy harvesting over the next reporting period can be formed. Even considering supercapacitor leakage, the schedule produced is still useful over multiple periods, as the energy loss to leakage should be similar across all nodes, i.e., only related to the duration between reporting rounds. We note that we can run the algorithm in [12] for as many iterations as we desire and always are able to return a valid schedule. This allows us to potentially offload the schedule computation to a more capable cloud machine as the network size increases.

Therefore, we adapt this existing algorithm to form the core of our scheduler. Since this schedule requires the current energy levels of our nodes, we must develop a way to predict these values. Most of our experimentation with different schedulers is done to test various prediction algorithms for the node energies. In addition, we also explore modifications to the load function in order to take into account energy harvesting. Since this algorithm produces a static schedule, we attempt to improve the schedule after each reporting period in order to take into account any energy level changes that may have occurred. We consider four alternatives to do so:

\subsection{LL - Oracle (LL-O)}

This particular variant uses an oracle which gives the exact current energy levels of each node in the network. This is done to provide a ceiling for the performance of the maximum lifetime network scheduling algorithm when operating with perfect knowledge of the energy situation in the network.

In this and the longest lifetime algorithms that follow, we start with a MINT schedule as our base schedule for improvement. Since the approximation algorithm only gives a tree and not a full schedule, we greedily build a schedule on top of the tree using the same heuristic as MINT. We select nodes to add to the schedule based on the number of candidates they interfere with, and greedily add as many as we can to that iteration of the schedule. We continue this process until the full tree has been scheduled.

\subsection{LL - Local Aggregate Information (LL-L)}

This variant of the longest lifetime scheduler uses local network information aggregated as part of our report to predict the node energies. During each reporting cycle, each node takes an average of its input power from the harvester. Then, during report aggregation, we aggregate the minimum average across all nodes (piggybacked along the actual data being aggregated). This gives us a pessimistic view of the expected energy harvested over the entire network that we can use to predict the available energy at the next reporting period.

Note that this approach does not differentiate between nodes in the network. That is, we predict all nodes to harvest the same amount of energy. The differences in energy then are solely a function of the last schedule that was used. Nodes will have expended different amounts of energy based on the number of message receptions at that node. 


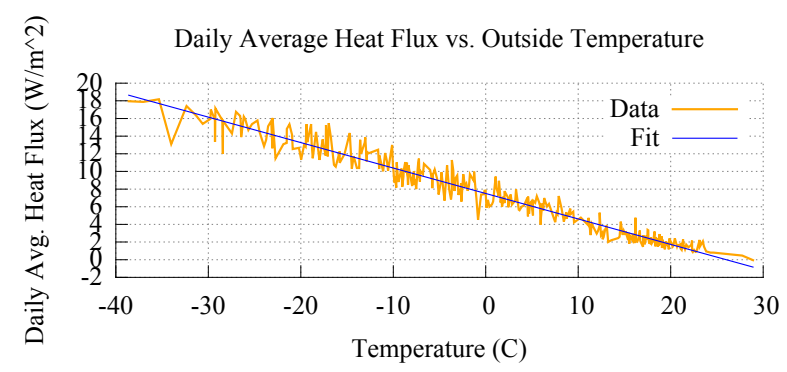

(a) Fit against local air temperature.

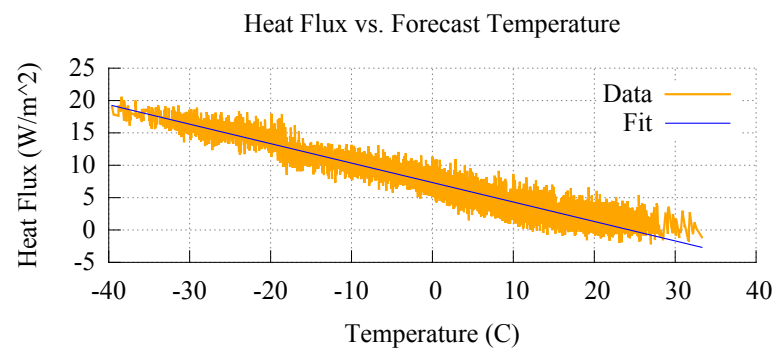

(b) Fit against forecast temperature.

Figure 1: Linear model fit of heat flux based on the 2013 dataset.

\subsection{LL - Local Air Temperature (LL-A)}

We also explored enhancing our energy predictions via additional information sources specific to the harvesting source used. One such attempt was to use the sensed outdoor air temperature to predict the next daily average heat flux for each apartment. The mapping from outdoor temperature to average daily heat flux is accomplished through a linear model that fits the heat flow to outside air temperature over a one year data set. Figure 1.a shows the combined data and fit for all apartments from the studied dataset during the calendar year 2013. We see that the data is quite noisy. We use this daily average to compute the expected node energies that will be available for the next reporting period when scheduling.

\subsection{LL - Weather Forecast (LL-F)}

Similar to LL-A, we attempt to predict the available node energy levels at report time. For this scheduler, we use the upcoming 24 hour forecast to inform the predictor. This forecast information is taken from historical Environment Canada weather data that was recorded at the Fort McMurray airport [1]. Using the heat flux data from each apartment, we fit a linear model of the forecast temperature to the hourly average power flux. Figure 1.b shows the combined data and fit for all apartments from calendar year 2013. We see that the data does exhibit some linearity, but is quite noisy. We use these hourly averages to compute the expected node energies that will be available for the next reporting period when scheduling.

This last scheduling algorithm represents an attempt at edge "fogification" by enhancing the edge-node behavior (scheduling) by utilizing information services external to the network. Though we assume that the forecast information is always available, it could be the case that we would need to fall back to either the local air temperature of aggregate information algorithm in the case of the external network becoming unreachable.

\section{PERFORMANCE STUDY}

As noted earlier we use the data from calendar year to evaluate the schedule construction and the associated harvested energy predictions. Initially, all energy buffers are assumed depleted. We then evolve the power model over time, making use of the heat flux data to inform the harvesting model. At each time step, we update the model with the harvested energy, until we reach the end of the reporting period. At each reporting period, we simulate the communication between nodes and then report on the results. If needed, the sink will then generate a new communication schedule for the next reporting period.

\subsection{Model Parameters}

For energy harvesting estimates, we will model thermoelectric generators from the heat flow passing through the apartment exterior walls. The heat flow data we use were collected by a sensor placed on a stud within the apartment wall.

For our data completeness reporting algorithm, we use a report resolution of 32, and a maximum lifetime of 4 . We calculate the message size using C++'s sizeof() operator to determine the size of the data completeness report in bytes. We define our actual data aggregation payload as a histogram with ten bins, but do not actually simulate the payload. Instead, we only use it to calculate the overall size of the message when combined with the completeness report.

We use a relatively modest thermoelectric generator with an area of $16 \mathrm{~cm}^{2}$ and an efficiency of 5\%. For the energy buffer, we model our supercapacitors after the Maxwell HC series of supercapacitors [2]. We use a supercapacitor with a $1 F$ capacitance, a leakage current of $0.006 \mathrm{~mA}$, and a maximum energy capacity of $3.6 \mathrm{~J}$.

We model our communication controller after the Texas Instruments CC2530 [3]. In our simulations, we use a transmission speed of $250 \mathrm{kbps}$, a transmission current of $39.6 \mathrm{~mA}$, a reception current of $29.6 \mathrm{~mA}$, an operating voltage of $2.7 \mathrm{~V}$, and a cut-off voltage of $2.0 \mathrm{~V}$.

We assumed a fixed communication graph (Figure 2) where each apartment (node) communicates with the nodes on the same floor as well as those directly above or below. This is similar to other work which used the same data set as this project [7].

\subsection{Performance Results}

Our main focus will be on a set of experiments which were run using a 24 hour reporting period/round. We will consider the following aspects of the scheduling algorithms: 


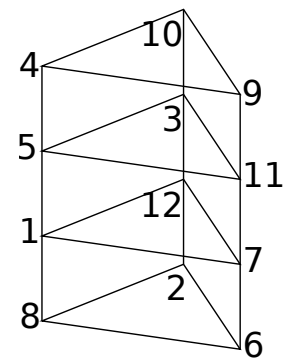

Figure 2: Simulated topology - node 1 is the sink.

- How often do we see sensor or communication failures due to node non-operability?

- How late do messages arrive at the sink, and how much data is lost?

- How accurate are our energy predictions?

- How do the schedules compare in terms of length and node degrees?

We also explored varying the node duty cycles as well as modifying the node load function to take into account energy harvesting.

6.2.1 Sensor Failures. Our rates of sensor data collection misses match closely to the overall performance of each scheduling algorithm. Figure 3 shows the rate of sensor data collection misses for each algorithm. Figure 4 shows the rate of communication failure for each algorithm, besides the oracle (LL-O) and local aggregate (LL-L) algorithms, which we omit as they had only 1 and 2 misses respectively.

We first notice that the local aggregate algorithm achieves performance very close to that of the oracle. These two algorithms see some sensor misses at the very start of the simulation, due to the energy buffers starting empty. We then see that once the controllers become operational they never experience energy depletion.

On the other hand, we see that the MINT algorithm performs poorly overall. The reason for this is that while the MINT algorithm produces a short schedule, the node degrees are not balanced. This leads to two nodes becoming overloaded and unable to forward data towards the sink after enough messages have been received. When we examined the node voltages closely, we noted that, once a day, at reporting time, the voltage drops below the communication controller operational voltage, causing communication and sensing failures until the node has harvested enough to become operational again. This leads to an order of magnitude more failures when using MINT as compared to the other scheduling algorithms.

Somewhat contrary to what we were initially expecting, both the local air temperature and weather forecast scheduling algorithms perform relatively poorly than the algorithm based on local aggregate information. The weather forecast based algorithm (LL-F) seems to have more failures than the local air temperature (LL-A) algorithm. While both algorithms perform better than MINT, they do not approach the performance ceiling (LL-O). In the following sections, we will drill deeper into why this is the case.

6.2.2 Message Latency and Completeness. In addition to the raw number of failures, we are also interested in seeing if our data completeness aggregation algorithm allows us to recover additional

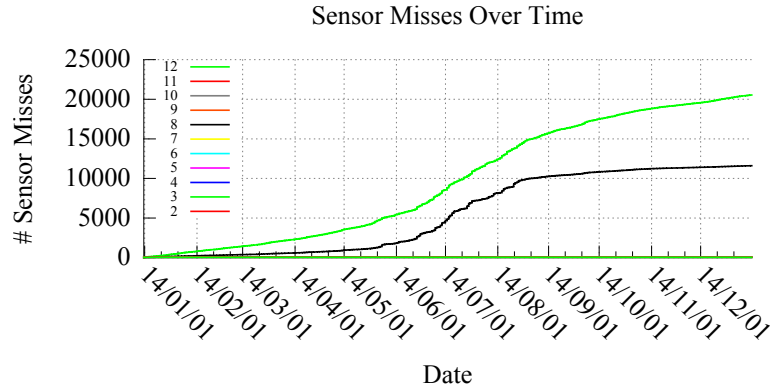

(a) MINT

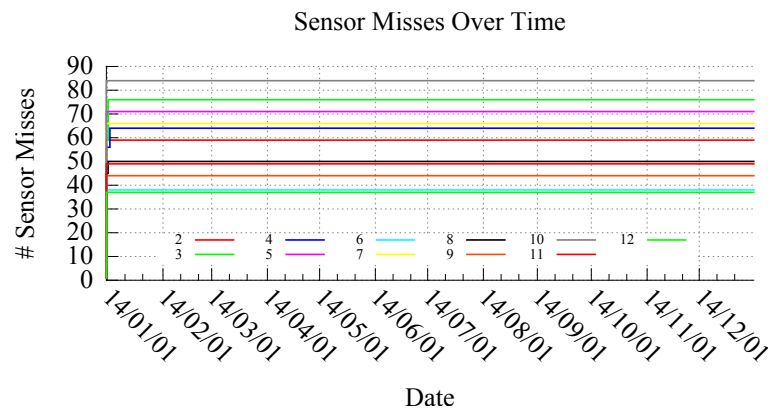

(b) LL-O

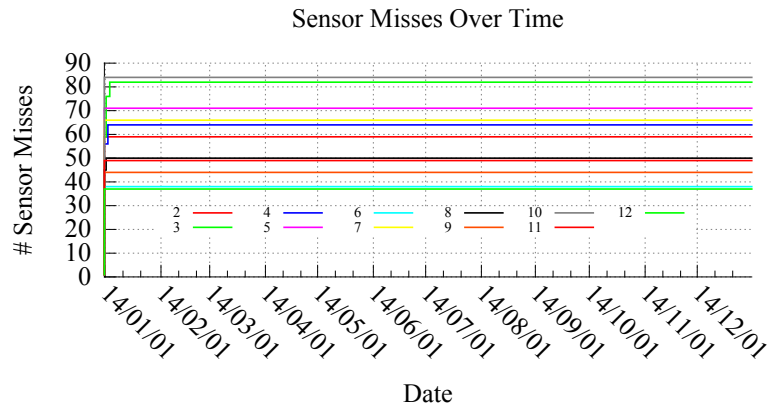

(c) LL-L

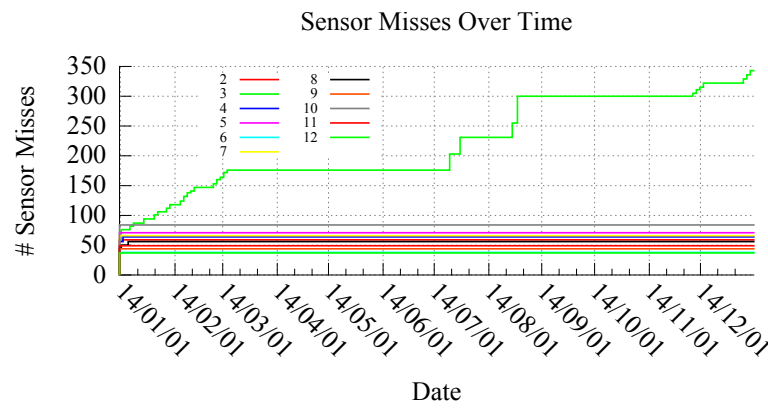

(d) LL-A

Figure 3: Cumulative sensor collection misses for each scheduling scheme. 


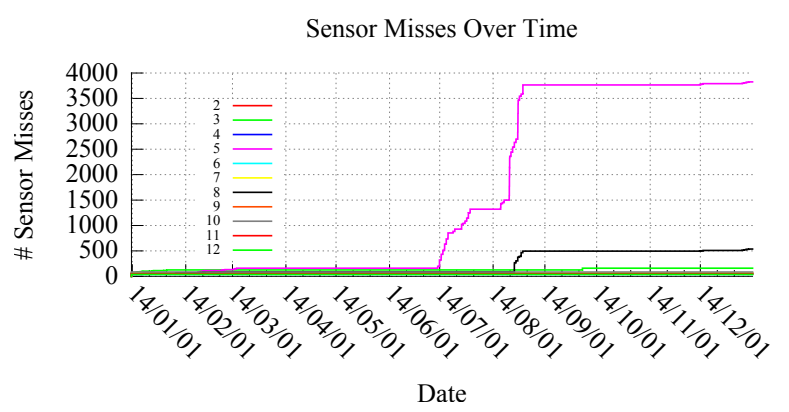

(e) LL-F

Figure 3: Cumulative sensor collection misses for each scheduling scheme. (cont'd)

\begin{tabular}{|l|l|l|l|l|l|}
\hline Algorithm & 1 & 2 & 3 & 4 & $\infty$ \\
\hline MINT & 728 & 0 & 0 & 0 & 3240 \\
\hline LL-O & 3991 & 13 & 0 & 0 & 0 \\
\hline LL-L & 3988 & 16 & 0 & 0 & 0 \\
\hline LL-A & 3847 & 151 & 6 & 0 & 0 \\
\hline LL-F & 3648 & 103 & 75 & 62 & 116 \\
\hline
\end{tabular}

Table 1: Number of reporting periods with latency to receive aggregate at the sink equal equal to $1,2,3$, and 4 rounds. Latency more than 4 rounds is equivalent to infinity.

information at the sink after a communication failure. Given that our simulation is running for 364 reporting periods (reporting period equal to one day) and that there are 11 non-sink nodes, we expect a total of $364 * 11=4004$ messages to reach the sink. Table 1 provides a summary of our results. Note that aggregated data that are received within one round are guaranteed to be exact, as no sub-sampling has been applied to them. Equally, the more the rounds until their reception, the less the potential accuracy as sub-sampling may have been repeatedly applied.

We find that the performance of each algorithm matches our expectations based on the observed communication failures from the previous section. We see that the MINT algorithm drops many messages. The reason for this is that in the network topology, all but two of the nodes are rooted behind nodes 3 and 8 . This results in energy depletion at those two nodes resulting in the loss of data from 9 of the 11 non-sink apartments. This further serves to reinforce the need for load balancing between the nodes, as failures at a few nodes close to the sink can result in not receiving data from a large portion of the network.

Overall, we see that LL-O (oracle-based) and LL-L (local aggregate information) algorithms receive the vast majority of messages in the same reporting period in which they were generated, with a few stragglers coming in the period directly afterwards. As the performance degrades by switching to the LL-A (air temperature) and LL-F (weather forecast) algorithms, we see an increasing number of messages are delayed, to the point where we begin to drop

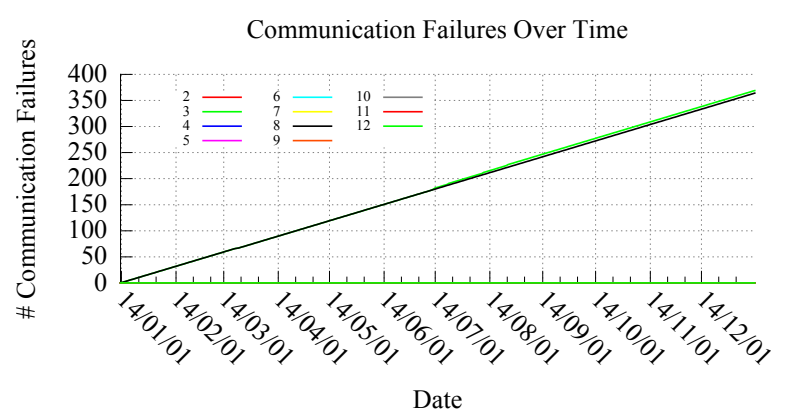

(a) MINT

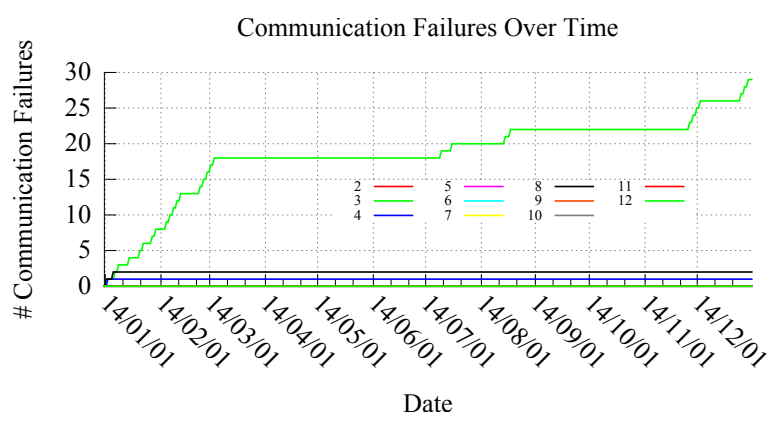

(b) LL-A

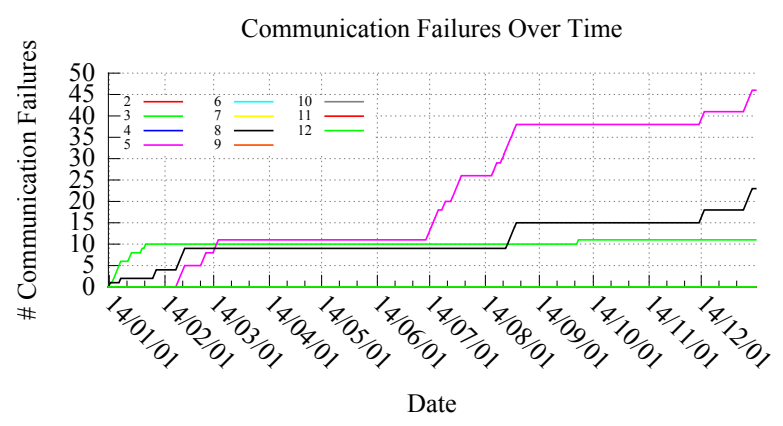

(c) LL-F

Figure 4: Cumulative sensor communication failures.

some messages when we exceed a latency of 4 rounds. Although these two algorithms show worse performance, they also serve to show that our data completeness aggregation algorithm is working as intended. As more communication failures occur in the network, we gracefully degrade in the quality of the data received at the sink. Indeed, we are also capable of eventually recovering most of the messages at the sink, rather than dropping them immediately. This is a good sign that our algorithm can handle temporary network faults which might delay message transmission for a small number of rounds. 


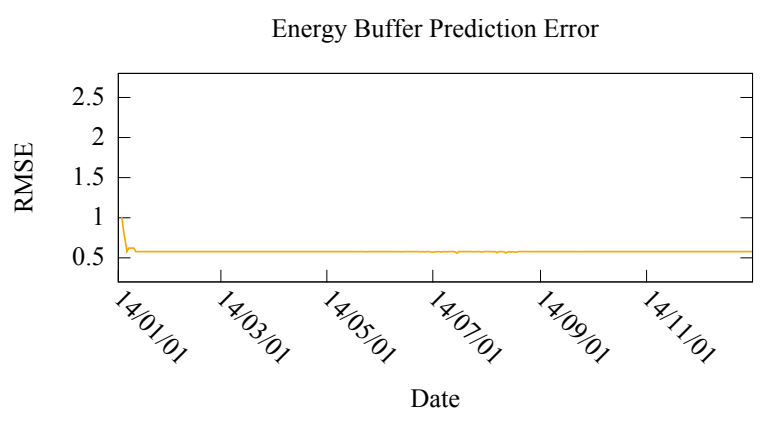

(a) LL-L

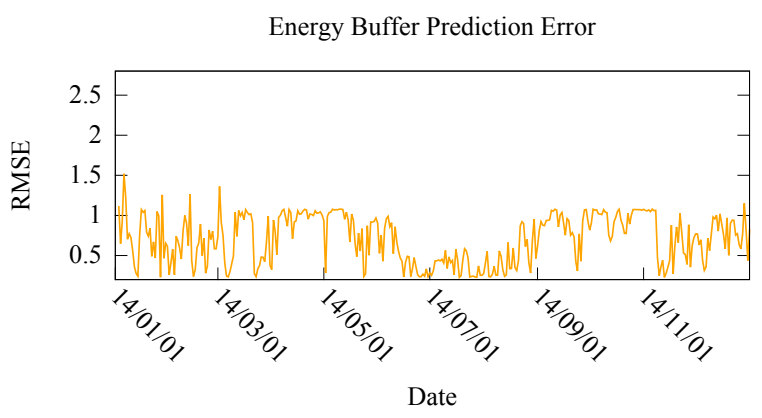

(b) LL-A

Energy Buffer Prediction Error

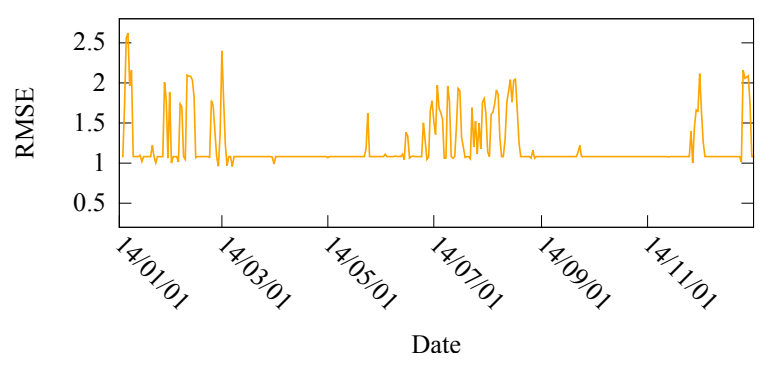

(c) LL-F

Figure 5: Energy prediction RMSE.

6.2.3 Energy Prediction. To further explore why we observed performance degradations when using the air temperature and weather forecast predictors, we calculated the error in energy prediction at each reporting cycle. Figure 5 shows the root mean squared error of the energy predictions across all nodes at each reporting cycle.

We observe that the local aggregate information provides quite accurate results for the energy prediction, with the RMSE hovering near $\sim 0.57$. For comparison, the energy buffer capacity is $3.6 \mathrm{~J}$. We see the error in the air temperature predictor vary quite wildly between an error of approximately 0.2 to 1.5 . This suggests that

\begin{tabular}{|l|l|l|l|}
\hline Algorithm & $\begin{array}{l}\text { Average } \\
\text { Length }\end{array}$ & $\begin{array}{l}\text { Maximum } \\
\text { Node Degree }\end{array}$ & $\begin{array}{l}\text { Number of } \\
\text { Changes }\end{array}$ \\
\hline MINT & 5 & 4 & 0 \\
\hline LL-O & 7.89 & 2.00 & 364 \\
\hline LL-L & 7.01 & 2.01 & 364 \\
\hline LL-A & 8.75 & 2.09 & 60 \\
\hline LL-F & 7.98 & 2.16 & 37 \\
\hline
\end{tabular}

Table 2: Characteristics of the produced schedules in terms of, length of schedule, maximum node degree, number of changes to the schedule over one year (daily data collection round).

while this predictor is inaccurate overall, it may be possible to improve it to achieve a lower error bound. Lastly, we see that the weather forecast predictor has an error consistently above 1 , with higher spikes up to $\sim 2$. These spikes in inaccuracy match up in time with the sensor and communication failures that we observe in Figures 3e and 4c. This suggests that inaccuracy in the energy buffer prediction can lead directly to energy violations caused by the resulting schedule. Overall, we see that the performance of the scheduling algorithm correlates closely with the accuracy of the energy buffer prediction.

6.2.4 Schedule Characterization. Finally, we characterize the properties of the generated schedules. Specifically, we are interested in the average length of the schedules we constructed as well as the average maximum node degree of non-sink nodes. In addition, we count the number of times the schedule was modified between reporting periods in response to changing node energy predictions. Table 2 summarizes our results.

We see that while the MINT algorithm produces the shortest schedule, it does so at the expense of the maximum node degree. This results in some nodes becoming overloaded, which causes communication and sensing failures as we repeatedly deplete the nodes of energy. On the contrary, the other algorithms produce longer schedules but do a better job of balancing the node loads, which results in a lower number of energy violations. Overall, we see that small differences in the longest lifetime schedules can result in relatively large performance differences.

We notice that the oracle and local aggregate information schedulers end up modifying the schedule with each reporting period. This results in the load being distributed between different nodes on a per reporting period basis. Qualitatively, we observed that these algorithms demonstrated a behaviour where nodes which were initially leafs of the tree ended up becoming interior nodes in the next schedule, and vice-versa. This had the result of spreading the energy consumption between nodes, and not overloading any one node by repeatedly using it for many message receptions in back-to-back schedules.

This was different from the air temperature and weather forecast scheduling algorithms, which modified the schedule infrequently between reporting periods. This meant that nodes with higher loads were not cycled out in consecutive reporting periods, which likely contributed to the worse performance of these algorithms. This is also reflected in the average maximum node degrees, where 


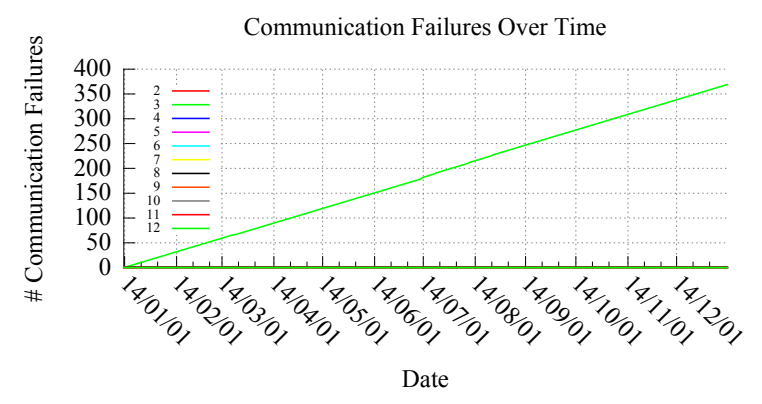

Figure 6: Cumulative communication failures for LL-L with modified load function.

we see a slight increase in node degree which suggests that the inaccurate energy predictions resulted in increased load on certain nodes which were not actually capable of supporting it.

6.2.5 Modifying the Load Function. Lastly, we explored modifying the node load function to take into account energy harvesting. We attempted to do so by adding the predicted harvested energy for each node to the node's load function as an offset to the communication cost. This resulted in the expression $f_{v}(x)=\frac{(x-1) E_{r x}+E_{t x}-H_{v}}{E_{v}}$, where $H_{v}$ is the predicted harvested energy for node $v$. The idea behind this was to change the load function to measure how many rounds of communication we could sustain with energy harvesting, as opposed to without. That is, if the communication costs minus the harvested energy had a residual cost, we would use this to count the number of reporting rounds until node energy depletion.

We tested this change using the local aggregate information algorithm with a 24 hour duty cycle, as this was the algorithm which exhibited the best performance. Unfortunately, our results show that this modification to the load function causes a performance degradation. Figure 6 shows the communication failures when running with the modified load algorithm.

We see that a bottleneck node is overloaded and continually failing. This results in that node dropping a total of 2160 network messages overall. This leads to a rather large performance degradation as compared to the unmodified load function. We see similar results when running with a 2 hour duty cycle.

We theorize that the harvesting offset too heavily obscures the current energy in the load function. For example, if a node has low energy now but expects a large harvest, we still shouldn not place additional load on it for the upcoming reporting period as that would cause communication failures. Therefore, our change seems to diminish the impact of the current node energies in the load function, which causes some nodes to take on more load than they really should. This results in those nodes becoming overloaded, which leads to communication failures and message drops. Put another way, we care mostly about the energy now when creating the schedule, since we need to respect the network energy constraints. Even if we expect to harvest lots of energy at those nodes in the future, overloading them according to their current energy will definitely result in communication failures.

\section{CONCLUSION}

We studied scheduling alternatives for energy harvesting aware aggregation convergecast. One novelty of our work is the use of data "completeness" metrics to capture the impact of energy depletion on the collected, aggregated, data when simple sub-sampling strategies are used to cope with the limited storage space of sensor nodes. Aggregation convergecast messages are also augmented to keep track of sub-sampled data reports in their aggregate form.

We have compared several different aggregation convergecast scheduling algorithms. In these algorithms data that were delivered in one round were guaranteed to be delivered without any loss. We found that algorithms which do not take node energies into account perform poorly, as the resulting schedule is not load balanced among the nodes. Overall, we found that predicting the node energies using pessimistic data gathered via our aggregation algorithm yielded the best results. Attempting to enhance the energy predictors using local air temperature and weather forecast information did not result in significant performance gains. Similarly, attempting to modify the node load function to take into account energy harvesting resulted in a performance regression.

In the future we will allow nodes to make localized decisions about deviating from the set schedule. This could perhaps be based on a small amount of additional communication in the network. One potential extension would be to allow nodes to share their energy levels with their immediate one-hop or two-hop neighbours before beginning reporting. Then, nodes could decide whether to locally modify the schedule based on this additional information.

\section{REFERENCES}

[1] (accessed April 22nd, 2018). Historical Climate Data. http://climate.weather.gc.ca/.

[2] (accessed April 22nd, 2018). Maxwell HC Series Supercapacitors. http://www. maxwell.com/images/documents/hcseries_ds_1013793-9.pdf.

[3] (accessed April 22nd, 2018). Texas Instruments CC2530. http://www.ti.com/lit/ds/ symlink/cc2530.pdf.

[4] X. Chen, X. Hu, and J. Zhu. 2005. Minimum data aggregation time problem in wireless sensor networks. Lecture Notes in Computer Science, Vol. 3794 LNCS. Institute of Applied Mathematics, Chinese Academy of Sciences.

[5] M. Jakob and I. Nikolaidis. 2016. A top-down aggregation convergecast schedule construction. In 2016 9th IFIP Wireless and Mobile Networking Conference (WMNC). 17-24. https://doi.org/10.1109/WMNC.2016.7543925

[6] A. Kollias and I. Nikolaidis. 2014. In-Wall Thermoelectric Harvesting for Wireless Sensor Networks. In SMARTGREENS 2014 - Proceedings of the 3rd International Conference on Smart Grids and Green IT Systems, Barcelona, Spain, 3-4 April, 2014. 213-221. https://doi.org/10.5220/0004864102130221

[7] A. Kollias and I. Nikolaidis. 2015. Seasonally aware routing for thermoelectric energy harvesting wireless sensor networks.. In SMARTGREENS 2015 - 4th International Conference on Smart Cities and Green ICT Systems, Proceedings. Computing Science Department, University of Alberta, 174-184.

[8] Z. G. Wan, Y. K. Tan, and C. Yuen. 2011. Review on energy harvesting and energy management for sustainable wireless sensor networks. In 2011 IEEE 13th International Conference on Communication Technology. 362-367. https: //doi.org/10.1109/ICCT.2011.6157897

[9] Y. Wu, S. Fahmy, and N. B. Shroff. 2008. On the Construction of a MaximumLifetime Data Gathering Tree in Sensor Networks: NP-Completeness and Approximation Algorithm. In IEEE INFOCOM 2008 - The 27th Conference on Computer Communications. https://doi.org/10.1109/INFOCOM.2008.80

[10] H. Yang and Y. Zhang. 2016. A task scheduling algorithm based on supercapacitor charge redistribution and energy harvesting for wireless sensor nodes. Fournal of Energy Storage 6 (2016), 186 - 194. https://doi.org/10.1016/j.est.2016.03.007

[11] T. Zhu, Z. Zhong, Y. Gu, T. He, and Z.-L. Zhang. 2009. Leakage-aware Energy Synchronization for Wireless Sensor Networks. In Proceedings of the 7th International Conference on Mobile Systems, Applications, and Services (MobiSys '09). ACM, New York, NY, USA, 319-332. https://doi.org/10.1145/1555816.1555849

[12] X. Zhu, G. Chen, S. Tang, X. Wu, and B. Chen. 2016. Fast Approximation Algorithm for Maximum Lifetime Aggregation Trees in Wireless Sensor Networks. INFORMS fournal on Computing 28, 3 (2016), 417 - 431. 